\title{
Defects in microvascular haemodynamics during prediabetes: contributor or epiphenomenon?
}

\author{
N. Wiernsperger \\ Lipha/INSERM U352, Villeurbanne, France
}

\begin{abstract}
Although diabetes is frequently characterized by microangiopathy, evidence accumulated over recent years suggests that most of these defects can be detected well before fasting hyperglycaemia is present. As will be explained, this observation applies even to prediabetic states that are not linked to defects in glucose tolerance, thereby suggesting that insulin resistance (or hyperinsulinaemia or both) are more causal than glycaemic control.

If so, the microcirculatory abnormalities have to be linked to both causal processes and possible consequences. The heterogeneity of the insulin-resistance state, which makes comparisons between patient groups questionable, and the technical difficulties inherent in researching microcirculation in relevant tissues are largely responsible for our lack of knowledge in this field. Nevertheless in this paper I examine possible hypotheses about origins and potential meaning of disturbances in microvascular haemodynamics during prediabetes.
\end{abstract}

\section{Microcirculation: main technological, anatomical and functional aspects}

To fully comprehend the subsequent analyses, a grasp of some fundamental characteristics of microcirculation that distinguishes it from large vessels is necessary.

\section{Technological specificities}

Microcirculation is difficult to access because of its dimensions and location and therefore requires

Corresponding author: N.Wiernsperger, Lipha/INSERM U352, INSA Building 406-20, avenue A.Einstein 69621 Villeurbanne, France adapted techniques. Most studies therefore deal with superficial tissues on which non-invasive techniques can be used: in humans, studies are thus limited to skin, finger nailfold or conjunctiva. This raises, however, the key question of relevance for other tissues when studying specific questions. Laser Doppler, hydrogen or thermoclearance and $\mathrm{Tc} p \mathrm{O}_{2}$ are the most local techniques in humans, of which only tissue $p \mathrm{O}_{2}$ has a sufficient resolution for true capillary flow. In animals, the ease of surgery allows the use of videomicroscopy to get access to deep tissues like the skeletal muscle, taking advantage of the thinness of some specific muscles (cremaster, spinotrapezius) for transillumination or epiillumination. Thereby vessels can be visualized in situ, diameters quantified and flow velocity measured. The use of fluorescent probes also permits an estimation of capillary and venular permeability as well as leucocyte adhesion.

\section{Anatomical and functional specificities}

Evidence is accumulating of major differences between characteristics of vascular cells (typically endothelium) according to tissues, vessel size and their functional role [1]. The very limited role of nitric oxide in small arterioles compared with arteries or even large arterioles [2] is a typical example of the importance of such aspects because most investigations deal with dilatatory reactions using nitric oxide-generating stimuli. Therefore, extrapolations of data from studies in larger vessels require extreme caution.

Microcirculation is the part of the vascular tree comprising vessels that range from first order arterioles (about $100 \mu \mathrm{m}$ diameter) to capillary venules, i.e. including terminal arterioles $(<15 \mu \mathrm{m})$ branching into true capillaries. Even arterioles of differing calibre (dependent on their location in the branching) 
behave differently: for example, precapillary arterioles in muscle are more richly innervated and more sensitive to noradrenaline than larger arterioles [3].

Arterioles participate in blood pressure regulation, maintain flow autoregulation and control the distribution of blood into the nutritive channels. Arterioles therefore maintain a pressure gradient across the vascular tree (from arteries to veins) and avoid development of capillary hyperperfusion/hypertension. This key function is achieved by a reactive constriction of arterioles to increases in shear stress, the so-called myogenic response [4]. Importantly, each arteriole can behave autonomically or in an integrated fashion within the network [5]. Defective myogenic responses lead to the well-known "haemodynamic" hypothesis of diabetic microangiopathy. Another constrictive reaction, the venoarteriolar reflex, develops in response to increases in venous pressure which typically occurs upon postural changes (upright positioning). Very importantly arteriolar constriction governs the distribution of blood into capillary units by the so-called precapillary (or arteriolar) vasomotion, a cyclic contraction/dilatation of terminal arterioles which diverts blood alternatively into neighbouring groups of capillaries at a frequency of 5-10 a min [6]. This "economic" procedure induces local dynamic flow behaviour, which is not only more efficient than regular flow [7] but also keeps reserve channels available for recruitment of additional capillary modules when needed [8]. Microvascular permeability is another important determinant of nutrient supply to target tissues, with pathological consequences whether too low (barrier) or exaggerated (leakage). In muscle the blood to tissue barrier is relatively tight so that the transfer of most nutrients (including glucose and insulin) is regulated by controlling transport processes.

These brief descriptions highlight some particular features of microcirculation: (1) microflow is tightly regulated by specific, local mechanisms of neuronal, humoral and metabolic origin and thus the microvascular bed must clearly be viewed as an autonomic entity, (2) blood distribution through capillary modules is more important than the absolute flow volume and (3) vasoconstriction, in sharp contrast with large vessels, seems to be an important component of the regulation of nutritive flow [9].

\section{Microcirculation defects in prediabetes}

Prediabetes is considered here as situations of undeclared or declared insulin resistance, preceding the clinical defined diabetic state of pathological fasting hyperglycaemia. Obviously this definition already encompasses variable situations ranging from young offspring of diabetic parents to those patients with impaired fasting glycaemia (IFG) or glucose intolerance (IGT).

\section{Structural changes}

Microvascular defects at these early stages can be of structural or functional nature or both. Unfortunately very few data exist concerning structural modifications in prediabetes. Increasing evidence showing the frequent existence of microalbuminuria in such groups is suggestive of early defects in capillary permeability, hence in favour of structural changes in endothelium and capillary basement membrane. Such alterations have been found even in normotolerant relatives of diabetic parents [10] and more frequently than expected in kidney and retina of IFG patients [11]. An obvious determinant of microflow is the number of capillaries within a tissue. The very limited data on this variable are puzzling: rarefaction of capillaries has been reported in (even mild) hypertension, a common component of the metabolic syndrome. Surprisingly, an increase in muscle capillarization has, however, been consistently found in prediabetic patients [12]. A plausible explanation is that a transient state, as a tentative adaptation to compensate for inadequate substrate delivery, exists before diabetes develops, a hypothesis which supports the concept I present in this paper.

\section{Functional changes}

These changes are almost exclusively alterations in the reactivity of middle to large sized vessels to various stimuli. For reasons outlined earlier, they are more indicative than truly explanatory, because the information has mostly been acquired in tissues or vessel segments which are not necessarily representative of other areas. With few exceptions flow changes are measured by techniques which, in addition to their lack of relevance for true microcirculation, are open to interpretation problems [13].

Animal data. Despite the availability of genetic strains, only few studies have dealt with microcirculatory investigations in prediabetic animals. One has looked at the chronological evolution of the process in insulin-resistant, fructose-fed rats and found that they were first insulin resistant, then endothelium-dependent vasodilatation defects appeared and finally hypertension developed [14]. Precapillary vasomotion was impaired in our study in the muscle of such rats after acute hyperglycaemia $[15,16]$. The myogenic response was impaired in non-diabetic, galactosefed rats [17].

Human data. In IFG patients endothelium-dependent dilatation was blunted [18]. As already found in IGT patients, cutaneous vasodilatation to local heating was impaired in IFG patients and correlated with insulin but not glycaemia [19]. 
These abnormalities were even seen in normotolerant subjects: vasodilatation in skin was impaired in various groups of insulin-resistant patients at risk for diabetes [20] and one study reported short-term and delayed defects in endothelium-dependent vasodilatation in women who had suffered gestational diabetes [21].

In healthy offspring of diabetic parents, flow-induced dilatation was reduced [22]. In skin, maximum vasodilatation was not impaired but minimum vascular resistance correlated negatively with insulin sensitivity [23]. Similarly a positive correlation was found between forearm dilatation and metabolic clearance rate [24]. Of great note, offspring of parents suffering intense hyperdynamic circulation, another situation characterized by high BMI and glucose intolerance, were insulin resistant [25]. Although the causes are not known, abnormalities in distribution of cardiac output can be envisaged as one causative factor. In patients with chronic heart failure, reduced insulin sensitivity has been related to changes in muscle blood flow [26]. There is also a concept emerging that associates low birth weight with insulin resistance and diabetes: in such patients cutaneous vasodilatation was impaired [27]. Offspring of normal rats fed a saturated fat diet also had vascular dysfunctions [28]. The increase in adipose tissue blood flow that normally follows OGTT was absent in obesity [29]. Another study reported, however, increased vasoconstriction in obesity [30].

Altogether these examples emphasize the very important notion that microvascular disturbances can be present long before diabetes develops and even before clinical symptoms of insulin resistance are apparent. This raises the question of causality. Importantly these defects are common to differing metabolic situations sharing links with variables of insulin resistance. This suggests that microcirculatory disturbances are linked to insulin resistance itself rather than hyperinsulinaemia.

\section{Possible origins of microvascular dysfunction}

From the clinical examples described above it seems that the microcirculatory abnormalities can be acquired even early in life. We have almost no data about the mechanisms involved and must therefore speculate as to how this occurs. Certainly the recent discoveries firstly that cell-cell interactions in vessels are operating by cellular signalling very similar to those used by insulin receptors $[31,32]$ and secondly that most vasoactive substances, including nitric oxide, also interfere with glucose transport [33], raise the possibility that vascular and metabolic defects are closely linked, even from the absolute beginning. Obesity, which is so frequent in prediabetic patients, can initiate or aggravate small vessel reactivity de- fects through the liberation of cytokines and it is noteworthy that in relatives of diabetic patients a correlation was found with a subtype of C-reactive proteins, CRPhs [24]. Fasting hyperinsulinaemia is linked to impaired fibrinolysis and hypercoagulability in normotolerant offspring [34], possibly leading to microthrombosis as postulated for early retinopathy.

Increasing evidence shows that oxidative stress might be a common denominator of these various clinical states. Although oxidative stress is a wellknown player in hyperglycaemic situations, we know much less about prediabetic, non-hyperglycaemic states. The link between hyperinsulinaemia and oxidative stress can be viewed in both directions. In insulin-resistant, non-diabetic animals, increased insulin concentrations were found together with enhanced oxidative stress [35]. High insulin concentration, both in vitro and in vivo, modifies the profile of antioxidant enzymes [36]. Again here the insulin resistant state itself, rather than hyperinsulinaemia, could be responsible for the production of free radicals through an activation of NADH/NADPH oxidase [37]. Iron, through increased ferritin concentrations [38] and thrombin [39] are favouring factors for oxidative stress. In low birthweight infants, oxidant status was impaired [40].

Although inherited factors could exist, it is tempting to consider postprandial events as being responsible for the impairment in the control of the oxidative balance and its interference with the microcirculation. High-carbohydrate and high-fat meals generate oxidative stress through various mechanisms [41-43]. Glycoxidation is considered to be the main reaction and, in addition, foods and drinks can contain large amounts of advanced glycation end products (AGEs). The potential of glucose and other sugars to generate oxidative stress is well-known but also oxidized LDLs and, more so glycoxidized LDLs are potent oxidants [44]. The importance of postprandial metabolic abnormalities is increasingly recognized and recent studies highlighted the link between IGT and the development of cardiovascular diseases [45, 46]. Further, the suggestion was recently made that patients with IFG and concomitant glucose intolerance have higher microangiopathy incidence than those who are normotolerant. Further investigations suggested that this might have been completely due to the first meal of the day ("first-meal effect") [47]. It could then be speculated that some people at risk for diabetes might be unable to ensure long-term compensation of the daily repetitive peaks in postprandial oxidative stress. High insulin concentrations dilate vessels and increased shear stress liberates free radicals [48]. Whereas these probably have physiological significance in normal situations, exaggerated radical production in the presence of high postprandial insulin concentrations such as occurs in obesity could also be deleterious in the long term [49]. 
Oxidative stress cannot only cause insulin resistance in non-vascular tissues $[50,51]$ but also induce disturbances in vascular cells such as impaired vasomotor reactivity, leucocyte/erythrocyte adhesion and permeability, i.e. the typical changes observed in prediabetic patients. According to this concept, these well-known defects would then "simply" be further exaggerated by the chronic hyperglycaemia in established diabetes.

Sympathetic neurotransmitters can profoundly affect the perfusion routes in muscle. High sympathetic output can shift microflow to non-nutritive areas and evidence is accumulating for neuroendocrine perturbations, including raised sympathetic activity being at the origin of insulin resistance [52]. The same phenomenon can also be involved in postprandial periods when both insulin and glucose are increased (see below).

Several authors have proposed that transendothelial transport of insulin is another vascular component in insulin resistance [53]. Although very few data are available, resistance to antilipolytic insulin effects in vivo, (in contrast to in vitro) together with reduced insulin clearance was seen in relatives of subjects with Type II (non-insulin-dependent) diabetes mellitus [54, 55].

\section{Microcirculatory disturbances: generators of insulin resistance?}

The previous examples show that qualitatively and quantitatively the changes in microcirculation are similar in prediabetes and established diabetes. Obviously they will not lead to microangiopathies as such if glycaemia is controlled over the long term. Our hypothesis is, however, that they have the potential to induce or aggravate pre-existing insulin resistance. Clearly this concept of an involvement of flow in metabolic regulation is not new $[53,56,57]$ and much evidence exists for such links. Here I try not only to describe a rationale for this concept but also to explain why the debate [58], based on particular experimental conditions and techniques, has not solved and will not solve this important question. I hope, however, to provide convincing arguments that analysing the problem from the point of view of microcirculation could reconcile apparently discrepant findings and that only sophisticated investigations on microcirculation will be able in the future to confirm, or not, the validity of these hypotheses.

\section{"Flow" and glucose delivery/uptake}

The main role of insulin after its secretion in postprandial periods is to induce the storage of glucose as glycogen in peripheral tissues, mainly skeletal mus- cle. Clearly a main mechanism of action of insulin is the stimulation of GLUT-4 translocation and activation of glycogen synthase. A relatively large difference in glucose uptake has, however, consistently been found between incubated and perfused muscles. Provided that this was not due to systematic artefacts in the conservation of the samples, it must be envisaged that blood supply in situ is another player in the process. As stated before, it is of utmost importance to distinguish between flow as an increased quantity of blood flowing through an organ and phenomena such as redistribution of a constant blood volume in a circuit or recruitment of additional capillaries. Unfortunately most clinical techniques used to quantify flow cannot detect distribution changes and relatively large changes ( $>28 \%$ ) must occur to be seen with conventional techniques [59]. The capacity for capillary recruitment in muscles is, however, enormous, as judged from data in exercise. Augmenting the number of perfused capillaries by either dilating terminal arterioles or by stimulating the precapillary vasomotion are mechanisms that can be expected to provide nutrients to much more muscle fibres. Thereby a reduction in diffusion distance would be easily achieved, which is considerably more efficient than an increase in volume and velocity of blood flowing through a main feeding vessel. Note that GLUT-4s are preferentially co-localized with capillaries in muscles [60], a notion which fully agrees with an important role for microflow. Evidently increased flow can but will not necessarily increase glucose uptake, depending on how it is induced and where it is manifested [61]. Further support of this concept is the link between maximum forearm blood flow and insulin sensitivity in young, borderline hypertensive men [62] and even in normal people [63].

\section{Hemodynamic effects of insulin: controversies}

Insulin and flow. Recent years have generated much data showing vasodilating effects of insulin. This "old" observation was re-evaluated when researchers started to look at the possible part played by the haemodynamic action of the hormone in peripheral glucose uptake. This topic has been the subject of intense debates, including a lively controversy in this journal [58] but an objective analysis of the opposing arguments provides a mitigated impression. Several important reasons for this controversy are briefly exposed here.

As discussed, technical aspects are responsible for a large number of the discrepancies: (1) most methods measure essentially blood volume changes and cannot detect small subtle changes in flow distribution, (2) most measurements are done in the forearm, which could behave differently from the leg and (3) most importantly, almost all studies were done under 
conditions of the euglycaemic hyperinsulinaemic clamp. This is a crucial problem for interpretation of data because the euglycaemic clamp is not well suited for investigating this kind of question due to its highly unphysiological characteristics: (1) insulin normally does not increase without concomitant increases in glucose, (2) blood concentrations of insulin in clamps are usually too high and, moreover, non-pulsatile, (3) these high hormone concentrations increase sympathetic discharge and liberate endothelin, i.e. factors interfering with normal vascular and metabolic regulation. Finally, deep venous glucose concentrations lie in the severe hypoglycaemic range and it is not known how this might affect the cross-talk between venous and arterial vessels.

Of note, a direct comparison of global compared with local blood flow in humans provided puzzling findings: insulin increased limb flow (measured by venous occlusion plethysmography) but this increase was not seen in the muscles but in the subcutaneous fat [64]. Similarly, in animals, glucose uptake was not correlated with femoral blood flow but with the metabolism of the microflow tracer [65]. In contrast, using a new technique, it was found that physiological concentrations of insulin increased microvascular blood volume while not modifying forearm flow as measured by plethysmography [66]. The absence of correlation between local and global flow could thus be a major reason for discrepant findings.

In addition to the problem of using high insulin with normal glucose concentrations, the concentrations attained could also lead to erroneous interpretations because high insulin concentrations can, under some circumstances, produce vasoconstriction [67, $68]$ or enhance the constriction induced by noradrenaline [69]. It is thus conceivable that in a number of studies substances liberated concomitantly under clamp conditions induce opposite effects in vascular as opposed to extravascular tissues.

Several protocols aimed at looking at this relation used a reduction in flow. Under such conditions, higher glucose extraction could, however, compensate for lower delivery with no net change in glucose uptake.

Another aspect that supported the controversy was the chronology of events, i.e. whether glucose uptake or vasodilatation comes first. This question is biased by flow and metabolism usually being tightly coupled, so that flow augments when metabolic needs augment. It is therefore likely that the "late" dilatations observed after several hours of clamps simply reflect the long-term adaptation of flow to the increasing metabolic demand induced by long-lasting infusions of increased total amounts of insulin. In strong contrast, direct observations of muscle arterioles in situ by videomicroscopy showed relaxation as early as $10 \mathrm{~min}$ after subcutaneous insulin treatment [16].
Despite similar final concentrations, major differences were seen when local (intrabrachial or intrafemoral) and systemic insulin treatment was compared, indicating that when plasma insulin concentrations increase peripherally in physiological conditions several mechanisms will simultaneously come into play. Globally these studies have suffered from having little physiological relevance, in particular the use of "isolated" hyperinsulinaemia.

Insulin and microcirculatory flow. Very few studies have investigated this problem directly. Insulin was found to dilate arterioles of various sizes in different preparations both in vitro and in vivo. Terminal arterioles dilated in the muscle [16] and, most importantly, physiological insulin alone even recruited capillaries in rats [70] and, despite the limited sensitivity of positron emission tomography scanning this was verified even in humans [71].

Insulin combined with glucose. Because postprandial insulin secretion is induced by nutrients, mostly sugars, the most physiological condition is to study the combination of insulin with glucose. Surprisingly very few studies have used this approach. A further effect of glucose to increase leg blood flow has been reported [72]. In healthy humans a mixed meal induced a $36 \%$ constriction in skin microflow, which was blunted in diabetic subjects [73], whereas the indocyanine green technique could not detect a flow effect across the leg [74]. By contrast, an oral glucose load led to increases in cutaneous laser Doppler signals and tc $p \mathrm{O}_{2}$, a good indicator of increased capillary perfusion [75]. Although we did not measure flow as such, we found that, whereas terminal arterioles dilated and lost cyclic vasomotion with insulin alone, the same concentration of insulin when induced by peripheral glucose infusion resulted in an arteriolar constriction with concomitant stimulation of the vasomotion (Fig.1) [16]. Although we cannot completely rule out a contribution by raised osmolality, this seems unlikely because the latter normally dilates vessels. This effect was observed within 10-15 min after glucose infusion, suggesting a direct vasoactive effect rather than a phenomenon secondary to increased tissue metabolism.

These data are important because they show that mechanisms involved in two situations strictly similar in insulinaemia can be completely different, as already observed when different routes of application are compared. It emphasizes the importance of experimental conditions and proximity to physiological conditions when interpreting this kind of data.

High glucose concentrations induce vasoconstriction and probably this constrictor effect predominates over the relaxing effect of insulin. Another possibility is the control of arteriolar haemodynamics by the nervous system because both high insulin and glu- 


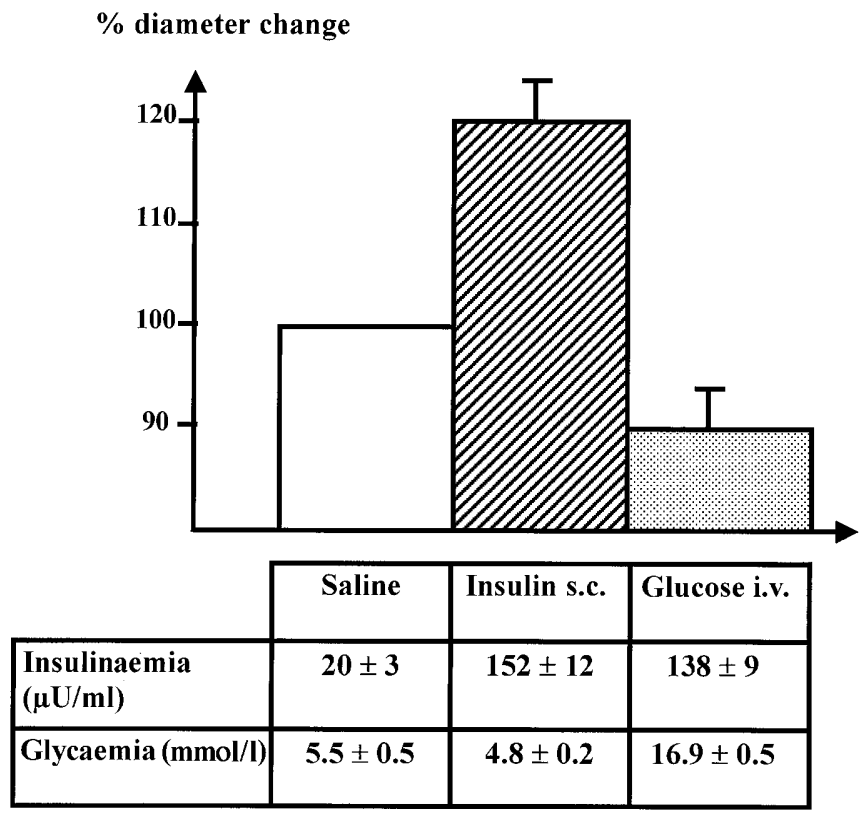

Fig. 1. Comparative change in muscle terminal arteriole diameter $15 \mathrm{~min}$ after infusion of either saline, insulin s. c. or glucose i.v. (from ref. 16)

cose stimulate the sympathetic nervous system, albeit through different routes and neurotransmitters. Because terminal arterioles are richly innervated they are a good target for such substances and might determine the ultimate microvascular reactivity.

Conversely reductions in flow achieved by approaches such as reflex vasoconstriction [61] or infusion of a specific microvascular constrictor [65] led to a corresponding decrease in glucose uptake, confirming the previous observation with nitric oxide blockade [56].

In our hypothesis, constriction of precapillary terminal arterioles with concomitant vasomotion normally govern the alternate filling of parallel running capillary units with whole blood. This permits the rapid supply to considerably more muscle fibres with no major change in absolute flow. For reasons stated previously, most techniques are unable to detect such changes, leading to erroneous conclusions about the involvement of "flow". As a typical illustration, a study combining three techniques for measuring flow at various depths found no increase in total forearm blood flow after oral glucose load, whereas flow doubled in subcutaneous tissue and decreased in skin [76], data reminiscent of effects seen with insulin [64]. Thus, redistribution can occur both within and between organs. If our data apply to human physiology, it means that flow redistribution or capillary recruitment or both are the mechanisms involved when either physiological insulin concentrations (through arteriolar dilatation) or the glucose/insulin mixture (through precapillary constriction and vasomotion) are studied. At postprandial levels, insulin alone could open arterioles and thereby perfuse most capillaries. At pathological plasma levels, insulin might, however, become deleterious to nutritive perfusion by either shunting blood towards other tissues or by acting as a vasoconstrictor if feeding vessels are injured. The physiological postprandial combination of moderately increased glucose and insulin, by contrast, could constrict terminal arterioles and activate cyclic vasomotion which, if occurring at the correct sites (sphincters?), control the constant redistribution of blood through truly nutritive channels.

Cardinal contributions have been that at least two microvascular circuits coexist in skeletal muscle, which can both be mobilized by precapillary vasoconstriction but their agonists differ and their location and function are different [56]. According to this concept, microflow can be either shunted within muscle and between muscle and other tissues (tendons, septae, fat, skin) or, on the contrary, directed through more nutritive capillaries.

It is thus proposed that disturbed microflow, in particular during postprandial phases, relies on inadequate constriction of the terminal arterioles. This defect might have different origins: excessive vasodilatation due to high insulin, lack of sensing of the precapillary receptors to their humoral or nervous stimuli, or lack of adequate effectiveness of the constricting effectors. By inference it means that during prediabetes any defect in this process, due to its daily repetitive nature over years, could act in concert with oxidative stress to eventually induce, but certainly worsen insulin resistance and favour the switch towards glucose intolerance and diabetes in those patients whose defence mechanisms could not sustain these effects over time. Supporting this notion, we showed in normoglycaemic insulin-resistant fructose-fed rats that, in contrast to controls (Fig. 2), i.v. glucose dilated arterioles without modifying vasomotion [15]. Moreover metformin, the only drug shown to constrict arterioles and induce/restore vasomotion, also improves insulin resistance in such animals, possibly through a link between both phenomena [34,77].

Recent findings showed that increasing non-nutritive flow also enhanced triglyceride hydrolysis, probably due to high enzyme activity in these circuits which mainly drain connective tissue and associated adipocytes [78]. Noteworthy is that first-degree relatives of Type II diabetic patients have postprandial hypertriglyceridaemia despite normal fasting triglyceride concentrations, even in the absence of glucose intolerance [79]. It is conceivable that defects in microflow distribution are a cause of this accumulation, considered to be a generator of insulin resistance [80]. 


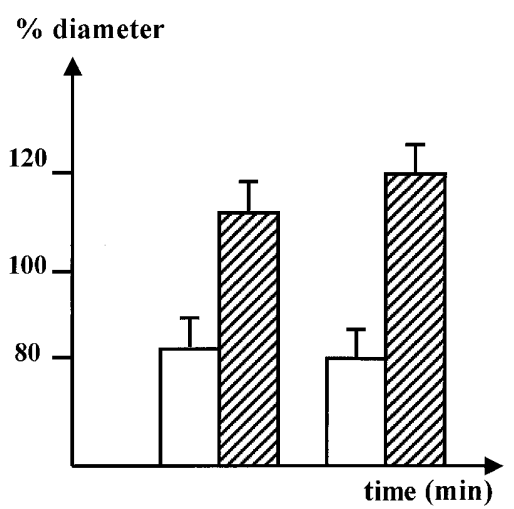

Fig. 2. Comparison between normal $(\square)$ and insulin resistant (fructose-fed) (VT) rats of the arteriolar haemodynamic reactions to short-term increases in glycaemia/insulinaemia (from ref. 15)

\section{Conclusion}

Paucity of direct data, technological problems and the extreme complexity of the interplay between so many vascular and metabolic factors (which further closely depend on substrate concentrations and integrity of cells) makes it very difficult to give a clear picture of the part played by microcirculation in prediabetes. Defects in microvascular reactivity are clearly present at this stage and, surprisingly, as pronounced as in established diabetes. These could be acquired as early as during the gestational period. According

Fig.3. Tentative integration of a microvascular component (thick lines) in the generation or aggravation of insulin resistance leading to diabetes and its complications

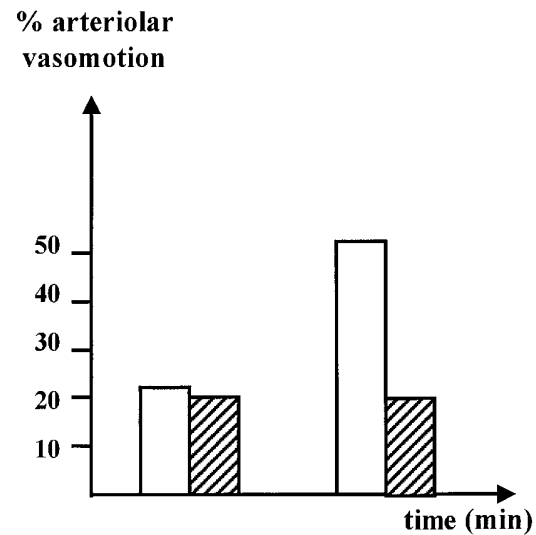

to our proposal (Fig. 3), defects which can be inherited, but are probably mainly acquired, develop in close association with insulin resistance. They include insulin resistance itself, hyperinsulinaemia, oxidative stress (probably generated by postprandial chemical reactions or by increased local shear stress in vessel walls) and sympathetic hyperactivity due to neuroendocrine disorders or to high circulating concentrations of insulin and glucose. Most links can operate in both directions. These factors will lead to loss of myogenic responses in arterioles, lack of appropriate vasoconstriction in terminal arterioles and capillary permeability. Thereby maldistribution of capillary flow will ensue, due to loss of precapillary vasomotion or to shunting towards non-nutritive channels or both. As a consequence, nutrient supply (in particular postprandial glucose delivery) will be impaired, leading to or reinforcing glucose intolerance. Once diabetes is established, they will act in conjunction with prevailing hyperglycaemia to develop microangiopathy. In our view this scenario, which requires intensive complementary investigations, reconciles contradictory views about an involvement of circulatory disturbances in metabolic regulation.

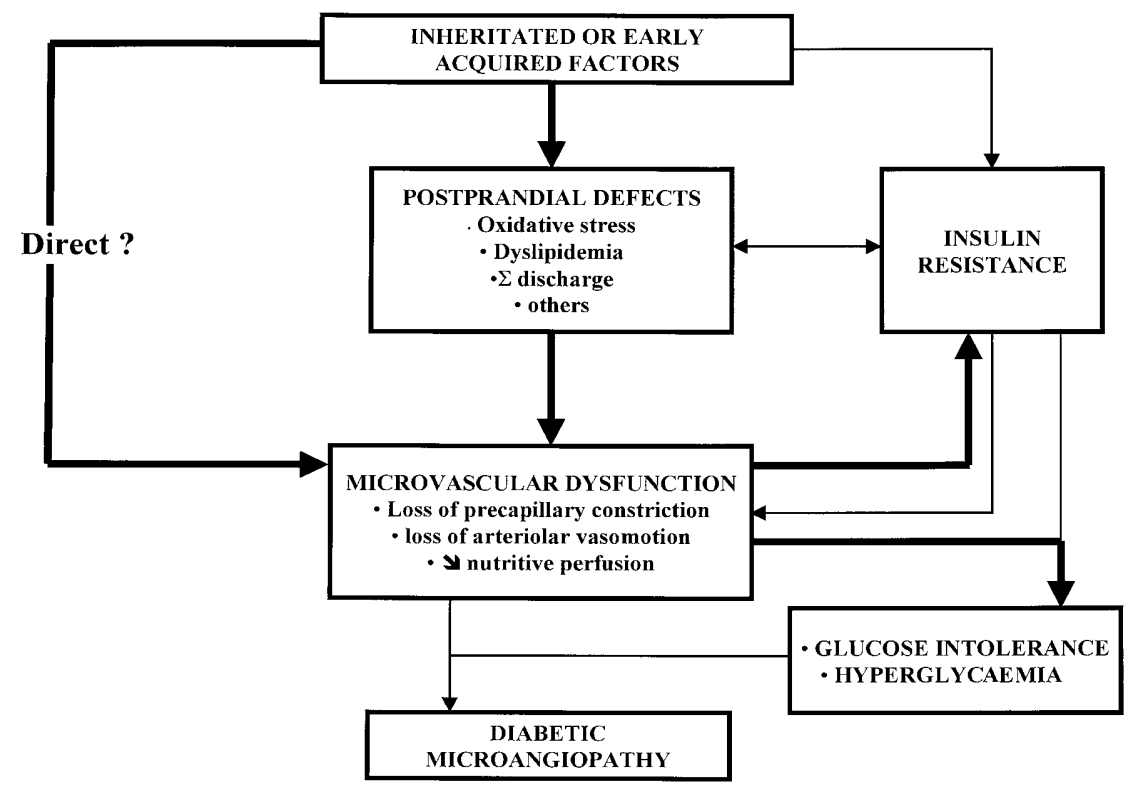




\section{References}

1. Brouland JP, Gilbert MA, Bonneau M, Pignaud G, Bal Dit Solier C, Drouet L (1999) Macro and microheterogeneity in normal endothelial cells: differential composition of luminal glycocalyx and functional implications. 6: 251-262

2. Pohl U, de Wit C, Gloe T (2000) Large arterioles in the control of blood flow: role of endothelium-dependent dilation. Acta Physiol Scand 168: 505-510

3. Rosell S (1980) Neuronal control of microvessels. Ann Rev Physiol 42: 359-371

4. Schubert R, Mulvany MJ (1999) The myogenic response: established facts and attractive hypotheses. Clin Sci (Colch) 96: 313-326

5. Cavalcanti S, Ursino M (1996) Chaotic oscillations in microvessel arterial networks. Ann Biomed Eng 24: 37-47

6. Intaglietta M (1990) Vasomotion and flowmotion: physiological mechanisms and clinical evidence. Vasc Med Rev 1: $101-112$

7. Parthimos D, Edwards DH, Griffith TM (1996) Comparison of chaotic and sinusoidal vasomotion in the regulation of microvascular flow. Cardiovasc Res 31: 388-399

8. Sweeney TE, Sarelius IH (1989) Arteriolar control of capillary cell flow in striated muscle. Circ Res 64: 112-120

9. Wiernsperger NF (2000) In defense of microvascular constriction in diabetes. Clin Hemorheol Microcirc (in press)

10. Feingold KR, Browner WS, Siperstein MD (1989) Prospective studies of muscle capillary basement membrane width in prediabetics. J Clin Endocrinol Metab 69: 784-789

11. Karunakaran S, Morris RJ, Aldington S et al. (1998) Microvascular and cardiovascular tissue damage over 3 years in the fasting hyperglycaemia study. Diabet Med 15 [Suppl 2]: P156 (Abstract)

12. Eriksson KF, Saltin B, Lindgärde F (1994) Increased skeletal muscle capillary density precedes diabetes development in men with impaired glucose tolerance. Diabetes 43: 805-808

13. Jorfeldt LS (1988) Measurement of skeletal muscle blood flow in humans: plethysmographic, bolus and continuous infusion technique. Am J Cardiol 62: 25E-29E

14. Katakam PV, Ujhelyi MR, Hoenig ME, Miller AW (1998) Endothelial dysfunction precedes hypertension in diet induced insulin resistance. Am J Physiol 275: R788-R792

15. Renaudin C, Michoud E, Lagarde M, Wiernsperger N (1997) Impaired microvascular responses to glucose in fructose-induced diabetic rats. Diabetologia 40 [Suppl 1]: A566 (Abstract)

16. Renaudin C, Michoud E, Rapin JR, Lagarde M, Wiernsperger N (1998) Hyperglycaemia modifies the reaction of microvessels to insulin in rat skeletal muscle. Diabetologia 41: $26-33$

17. Forster HG, Ter Wee PM, Takenaka T, Hohman TC, Epstein M (1994) Impairment of afferent arteriolar myogenic responsiveness in the galactose-fed rat. Proc Soc Exp Biol Med 206: 365-374

18. Vehkavaara S, Seppäla-Lindroos A, Westerbacka J, Groop PH, Yki-Järvinen H (1999) In vivo endothelial dysfunction characterizes patients with impaired fasting glucose. Diabetes Care 22: 2055-2060

19. Jaap AJ, Shore AC, Tooke JE (1997) Relationship of insulin resistance to microvascular dysfunction in subjects with fasting hyperglycaemia. Diabetologia 40: 238-243

20. Caballero AE, Arora S, Saouaf R et al. (1999) Microvascular and macrovascular reactivity is reduced in subjects at risk for type 2 diabetes. Diabetes 48: 1856-1862

21. Anastasiou E, Lekakis JP, Alevizaki M et al. (1998) Impaired endothelium-dependent vasodilatation in women with previous gestational diabetes. Diabetes Care 21: 2111-2115

22. Balletshofer BM, Rittig K, Enderle MD et al. (2000) Endothelial dysfunction is detectable in young normotensive first-degree relatives of subjects with type 2 diabetes in association with insulin resistance. Circulation 101: 1780-1784

23. Tooke JE, Lee BC, Humphreys JM, Hattersley AT, Shore AC (1999) Microvascular function and features of insulin resistance syndrome in healthy adults. Diabetologia 42 [Suppl 1]: A325 (Abstract)

24. Enderle MD, Beckmann P, Balletshofer B et al. (1999) Negative correlation of high sensitive CRP with insulin sensitivity and endothelial function in first degree relatives of Type II diabetics. Diabetologia 42 [Suppl 1]: A1-A330 (Abstract)

25. Palatini P, Vriz O, Nesbitt S et al. (1999) Parental hyperdynamic circulation predicts insulin resistance in offspring. Hypertension 33: 769-774

26. Houghton AR, Harrison M, Perry AJ, Evans AJ, Cowley AJ (1998) Endogenous insulin and insulin sensitivity. An important determinant of skeletal muscle blood flow in chronic heart failure? Eur Heart J 19: 476-480

27. Tooke JE, Goh KL (1999) Vascular function in Type 2 diabetes mellitus and pre-diabetes: the case for intrinsic endotheliopathy. Diabet Med 16: 710-715

28. Koukkou E, Ghosh P, Lowy C, Poston L (1998) Offspring of normal and diabetic rats fed saturated fat in pregnancy demonstrate vascular dysfunction. Circulation 98: 2899-2904

29. Jansson PA, Larsson A, Lönnroth PN (1998) Relationship between blood pressure, metabolic variables and blood flow in obese subjects with or without non-insulin-dependent diabetes mellitus. Eur J Clin Invest 28: 813-818

30. Nestel PJ, Yamashita T, Sasahara T et al. (1998) Control of the forearm microcirculation: interactions with measures of obesity and noradrenaline kinetics. Clin Sci (Colch) 95: 203-212

31. Bertuglia S, Colantuoni A (1998) Insulin-induced arteriolar dilation after tyrosine kinase and nitric oxide synthase inhibition in hamster cheek pouch microcirculation. J Vasc Res 35: 250-256

32. Zeng G, Nystrom FH, Ravichandran LV et al. (2000) Roles of insulin receptor, PI3-kinase, and Akt in insulinsignaling pathways related to production of nitric oxide in human vascular endothelial cells. Circulation 101: $1539-1545$

33. Shankar RR, Wu Y, Shen HQ, Zhu JS, Baron AD (2000) Mice with gene disruption of both endothelial and neuronal nitric oxide synthase exhibit insulin resistance. Diabetes 49: 684-687

34. Meigs JB, Mittleman MA, Nathan DM et al. (2000) Hyperinsulinaemia, hyperglycaemia, and impaired haemostasis. JAMA 283: 221-228

35. Faure P, Rossini E, Wiernsperger N, Richard MJ, Favier A, Halimi S (1999) An insulin sensitizer improves the free radical defense system potential and insulin sensitivity in high fructose-fed rats. Diabetes 48: 353-357

36. Pereira B, Rosa LF, Safi DA, Bechara EJ, Curi R (1995) Hormonal regulation of superoxide dismutase, catalase, and glutathione peroxidase activities in rat macrophages. Biochem Pharmacol 50: 2093-2098

37. Kashiwagi A, Shinozaki K, Nishio Y, Okamura T, Toda N, Kikkawa R (1999) Free radical production in endothelial cells as a pathogenetic factor for vascular dysfunction in the insulin resistance state. Diabetes Res Clin Pract 45: 199-203 
38. Ford ES, Cogswell ME (1999) Diabetes and serum ferritin concentration among U.S. adults. Diabetes Care 22: 1978-1983

39. Holland JA, Meyer JW, Chang MM, O’Donnell RW, Johnson DK, Ziegler LM (1998) Thrombin stimulated reactive oxygen species production in cultured human endothelial cells. Endothelium 6: 113-121

40. Friel JK, Ziegler EE, Widness JA, Jiang T, Belkhode SL, Rebouche CJ (1999) Antioxidant status and oxidant stress in very low birthweight infants during the first month of life. Free Radic Biol Med 27 [Suppl 1]: S121

41. Bakker SJ, Ijzerman RG, Teerlink T, Westerhoff HV, Gans RO, Heine RJ (2000) Cytosolic triglycerides and oxidative stress in central obesity: the missing link between excessive atherosclerosis, endothelial dysfunction, and beta-cell failure? Atherosclerosis 148: 17-21

42. Plotnick GD, Corretti MC, Vogel RA (1997) Effect of antioxidant vitamins on the transient impairment of endothelium dependent brachial artery vasoactivity following a single high-fat meal. JAMA 278: 1682-1686

43. Ceriello A (2000) The post-prandial state and cardiovascular disease: relevance to diabetes mellitus. Diabetes Metab Res Rev 16: 125-132

44. Galle J, Schneider R, Winner B et al. (1998) Glycoxidized LDL impair endothelial function more potently than oxidized LDL: role of enhanced oxidative stress. Atherosclerosis 138: 65-77

45. Lefebvre PJ, Scheen AJ (1998) The postprandial state and risk of cardiovascular disease. Diabet Med 15 [Suppl 4]: S63-S68

46. Tominaga M, Eguchi H, Manaka H, Igarashi K, Kato T, Sekikawa A (1999) Impaired glucose tolerance is a risk factor for cardiovascular disease, but not impaired fasting glucose. Diabetes Care 22: 920-924

47. Baron AD, Clark PR, Lazar C, Fineberg N (1999) Isolated postprandial hyperglycaemia may cause microangiopathy. A "first-meal effect" in patients with early type 2 diabetes mellitus. Diabetes 48 [Suppl 1] A123 (Abstract)

48. Howard AB, Alexander RW, Nerem RM, Griendling KK, Taylor WR (1997) Cyclic strain induces an oxidative stress in endothelial cells. Am J Physiol 272: C421-C427

49. Laurindo FR, de Almeida Pedro M, Barbeiro HV et al. (1994) Vascular Free Radical Release Ex vivo and in vivo evidence for a flow dependent endothelial mechanism. Circ Res 74: 700-709

50. Laight DW, Desai KM, Gopaul NK, Anggard EE, Carrier MJ (1999) Pro-oxidant challenge in vivo provokes the onset of NIDDM in the insulin resistant obese Zucker rat. $\mathrm{Br}$ J Pharmacol 128: 269-271

51. Paolisso G, Giugliano D (1996) Oxidative stress and insulin action: is there a relationship? Diabetologia 39: 357-363

52. Bjorntorp P (1999) Neuroendocrine perturbations as a cause of insulin resistance. Diabetes Metab Res Rev 15: 427-441

53. Wiernsperger N (2000) Metformin: intrinsic vasculoprotective properties. Diab Tech The 2: 259-272

54. Eriksson JW, Smith U, Waagstein F, Wysocki M, Jansson PA (1999) Glucose turnover and adipose tissue lipolysis are insulin-resistant in healthy relatives of type 2 diabetes patients: is cellular insulin resistance a secondary phenomenon? Diabetes 48: 1572-1578

55. Wascher TC, Wölkart G, Russel JC, Brunner F (2000) Delayed insulin transport across endothelium in insulin-resistant JCR: LA-cp rats. Diabetes 49: 803-809

56. Baron AD, Clark MG (1997) Role of blood flow in the regulation of muscle glucose uptake. Annu Rev Nutr 17: 487-499
57. Ganrot PO, Curman B, Kron B (1987) Type 2 diabetes. Primary vascular disorder with metabolic symptoms? Med Hypotheses 24: 77-86

58. Yki-Järvinen H, Utriainen T (1998) Insulin-induced vasodilatation: physiology or pharmacology? Diabetologia 41: 369-379

59. Bonadonna RC, Saccomani MP, Del Prato S, Bonora E, DeFronzo RA, Cobelli C (1998) Role of tissue specific blood flow and tissue recruitment in insulin-mediated glucose uptake of human skeletal muscle. Circulation 98: 234-241

60. Gaster M, Schroder HD, Handberg A, Beck-Nielsen B (1998) Colocalisation between human muscle capillaries and Muscle GLUT-4. Diabetes 47 [Suppl 1] 1169

61. Jamerson KA, Smith SD, Amerena JV, Grant E, Julius S (1994) Vasoconstriction with norepinephrine causes less forearm insulin resistance than a reflex sympathetic vasoconstriction. Hypertension 23: 1006-1011

62. Fossum E, Hoieggen A, Moan A, Rostrup M, Nordby G, Kjeldsen SE (1998) Relationship between insulin sensitivity and maximal forearm blood flow in young men. Hypertension 32: 838-843

63. Serné EH, Stehouwer CD, Ter Maaten JC et al. (1999) Microvascular function relates to insulin sensitivity and blood pressure in normal subjects. Circulation 99: 896-902

64. Rosdahl H, Lind L, Millgard J, Lithell H, Ungerstedt U, Henriksson J (1998) Effect of physiological hyperinsulinaemia on blood flow and interstitial glucose concentration in human skeletal muscle and adipose tissue studied by microdialysis. Diabetes 47: 1296-1301

65. Rattigan S, Clark MG, Barrett EJ (1999) Acute vasoconstriction induced insulin resistance in rat muscle in vivo. Diabetes 48: 564-569

66. Coggins M, Fasy E, Lindner J et al. (1999) Physiologic hyperinsulinaemia increases skeletal muscle microvascular blood volume in healthy humans. Diabetes 48 [Suppl 1]: 958 A220 Abstract

67. Schroeder CA, Chen YL, Messina EJ (1999) Inhibition of NO synthesis or endothelium removal reveals a vasoconstrictor effect of insulin on isolated arterioles. Am J Physiol 276: H815-H820

68. Villa E, Gonzalez-Albarran O, Rabano A, Garcia-Robles R (1999) Effects of hyperinsulinaemia on vascular blood flows in experimental obesity. J Steroid Biochem Mol Biol 69: 273-279

69. Van Veen S, Chang PC (1998) Modulation of vasoconstriction by insulin. J Hypertens 16: 1157-1164

70. Rattigan S, Clark MG, Barrett EJ (1197) Hemodynamic actions of insulin in rat skeletal muscle. Diabetes 46: $1381-1388$

71. Raitakari M, Knuuti MJ, Ruotsalainen U et al. (1995) Insulin increases blood volume in human skeletal muscle: studies using (150) CO and positron emission tomography. Am J Physiol 269: E1000-E1005

72. Cleland SJ, Petrie JR, Ueda S, Elliott HL, Connell JM (1999) Insulin-mediated vasodilation and glucose uptake are functionally linked in humans. Hypertension 33: 554-558

73. Rossi M, Lall K, Standfield N, Dornhorst A (1998) Impaired vasoconstriction of peripheral cutaneous blood flow in type 1 diabetic patients following food ingestion. Diabet Med 15: 463-466

74. Hernandez Mijares A, Jensen MD (1995) Contribution of blood flow to leg glucose uptake during a mixed meal. Diabetes 44: 1165-1169

75. Forst T, Kunt T, Pohlmann T et al. (1998) Microvascular skin blood flow following the ingestion of $75 \mathrm{~g}$ glucose in 
healthy individuals. Exp Clin Endocrinol Diabetes 106: 454-459

76. Bülow J, Astrup A, Christensen NJ, Kastrup J (1987) Blood flow in skin, subcutaneous adipose tissue and skeletal muscle in the forearm of normal man during an oral glucose load. Acta Physiol Scand 130: 657-661

77. Wiernsperger N (1994) Vascular defects in the aetiology of peripheral insulin resistance in diabetes. A critical review of hypotheses and facts. Diabetes Metab Rev 3: 287-307

78. Clerk LH, Smith ME, Rattigan S, Clark MG (2000) Increased chylomicron triglyceride hydrolysis by connective tissue flow in perfused rat hindlimb. Implications for lipid storage. J Lipid Res 41: 329-335

79. Axelsen M, Smith U, Eriksson JW, Taskinen MR, Jansson PA (1999) Postprandial hypertriglyceridemia and insulin resistance in normoglycaemic first-degree relatives of patients with type 2 diabetes. Ann Intern Med 131: 27-31

80. Perseghin G, Scifo P, De Cobelli F et al. (1999) Intramyocellular triglyceride content is a determinant of in vivo insulin resistance in humans. Diabetes 48: 1600-1606 